\title{
Effect of $\mathrm{CpG}$ methylation on $\mathrm{RAG1/RAG2}$ reactivity: implications of direct and indirect mechanisms for controlling V(D)] cleavage
}

\author{
Hiroshi Nakase ${ }^{1,2}$, YousukeTakahama ${ }^{1,2} \&$ Yoshiko Akamatsu ${ }^{1++}$ \\ ${ }^{1}$ Institutefor Genome Research, University of Tokushima, Tokushima, Japan, and 2RI KEN Research Center for Allergy \\ and Immunology, Tokushima, Japan
}

\begin{abstract}
It has been suggested that DNA methylation/demethylation is involved in regulating $V(D)$ J rearrangement. Although methylated DNA is thought to induce an inaccessible chromatin structure, it is unclear whether DNA methylation can directly control V(D)J recombination independently of chromatin structure. In this study, we tested whether DNA methylation directly affects the reactivity of the RAG 1/RAG 2 complex. Specific methylation within the heptamer of the recombination signal sequences (RSS) markedly reduced V(D)J cleavage without inhibiting RAG 1/RAG 2-DNA complex formation. By contrast, methylation at other positions around the RSS did not affect the reactivity of the RAG proteins. The presence of a methyl-CpG binding-domain protein inhibited the binding of the RAG 1/RAG 2 complex to all the methylated CpG sites that were tested. 0 ur findings suggest that DNA methylation around the RSS may have a previously unexpected function in regulating $V(D) J$ recombination by directly inhibiting $V(D)$ J cleavage, in addition to its general function of inducing an inaccessible chromatin configuration.

EM BO reports 4, 774-780 (2003)

doi:10.1038/sj.embor.embor904
\end{abstract}

\section{INTRO DUCTION}

During lymphocyte development, antigen receptor genes are assembled from V, D and J gene segments to form functional exons of their variable region. This process, termed $V(D)$ J recombination, is initiated by the lymphoid-specific proteins, RAG 1 and RAG2 (Schatz et al., 1989; O ettinger et al., 1990). RAG 1 and RAG2 form a stable complex to recognize the well-conserved recombination signal sequences (RSSs) that flank each antigenreceptor gene segment. RSSs consist of highly conserved heptamer and nonamer regions that are separated by a less-conserved

\footnotetext{
IInstitutefor Genome Research, University of Tokushima, Tokushima 770-8503, Japan 2RIKEN Research Center for Allergy and Immunology, Tokushima 770-8503, Japan +Corresponding author. Tel: +1 510574 1609; Fax: +1 510574 1500;

E-mail: yakamatsu@pdl.com

tPresent address: Protein Design Labs, Inc., 34801 Campus Drive, Fremont, California 94555, USA
}

Received 7 January 2003; revised 30 May 2003; accepted 18 June 2003 12-bp or 23-bp spacer (12-RSS and 23-RSS, respectively). Efficient recombination occurs only between 12-RSSs and 23RSSs, and this is known as the $12 / 23$ rule, because a doublestranded break (DSB) is catalysed preferentially within the paired complex (PC), in which a 12-RSS and a 23-RSS are synapsed (coupled cleavage). The RAG 1/RAG 2 complex mediates DSB formation in a two-step process. First, a nick is introduced at the border between the coding sequence and the RSS heptamer on its sense strand; next, the 3' OH of the nick attacks the opposite (antisense) strand to form a hairpin coding end and a blunt signal end. Purified RAG 1 and RAG 2 proteins are sufficient to obey the 12/23 rule to form the $P C$ and perform coupled cleavage, supported by the high-mobility group protein HMG1, or HMG2. These RAGmediated reactions are the first step in the several processes that are involved in $V(D)$ ) recombination (Gellert, 2002).

$\mathrm{V}(\mathrm{D}) \mathrm{J}$ recombination is highly ordered and is controlled at several levels, including the levels of tissue- and site-specificity, lymphocyte developmental stage and allelic exclusion (Bassing et al., 2002). To account for the different levels of regulation, an accessibility model has been proposed whereby germline RSS sites, which are inaccessible to the recombination machinery due to chromatin structure or modification, must be actively opened before recombination takes place (Blackwell \& Alt, 1989). O ne of the potential mechanisms of controlling accessibility is DNA methylation. It has been shown that CPG methylation has an important function in regulating transcription and chromatin structure in several genes (Bird \& Wolffe, 1999; Wade, 2001). DN A methylation is known to repress gene expression directly by impeding the binding of trans-acting factors, and indirectly by the recruitment of histone deacetylases (HDACs) through methyl CpG $(\mathrm{mCpG})$ binding-domain (M BD) family proteins (Bird \& Wolffe, 1999). Current studies suggest a model in which MBD family proteins function as anchors on methylated DNA, recruiting accessory proteins such as HDACs that are able to modulate chromatin structure and the transcriptional activity of genes (Wade, 2001).

Differences in methylation status are also correlated with antigenreceptor gene rearrangement and expression. For example, immunoglobulin and T-cell receptor (TCR) loci are highly methylated before $V(D)$ J recombination and undergo demethylation concomitantly with gene rearrangement (M ostoslavsky \& Bergman, 1997). 
The demethylation at the $\lg \kappa$ locus is monoallelic, suggesting a function in the maintenance of allelic exclusion (Mostoslavsky et al., 1998). At the TCR- $\beta$ locus, deletion of the $D \beta 1$ promoter enhances methylation at proximal sites and results in reduced recombination (W hitehurst et al., 2000). Although demethylation alone is not sufficient to activate $V(D)$ ] rearrangement (Cherry et al., 2000), these studies strongly suggest that DNA methylation is important in the regulation of antigen-receptor genes in vivo.
It has been shown that a $\mathrm{CpG}$ site in the heptamer of broken signal ends derived from the 3' D $\beta 1$ RSS are completely demethylated in mouse, suggesting that methylation of this site is incompatible with $\mathrm{V}(\mathrm{D})$ J cleavage (W hitehurst et al., 2000). This heptamer sequence is present in the 12-RSSs of the DQ 52, Vк24C, D $\delta 2$ and J $\delta 2$ gene segments in mouse antigen-receptor genes, and is also commonly found in the $D_{H}$ genes of chicken and rabbit. As potential methylation sites are localized in many RSSs, as well as in their flanking coding

Table $1 \mid$ Oligonucleotides used in this study

\begin{tabular}{|c|c|}
\hline Oligonucleotide & Sequence \\
\hline \multicolumn{2}{|l|}{ 12-RSS standard } \\
\hline HN 100 & 5'-CTGCAGGTCAACCTGCACAGTGCTACAGACTGGA_ACAAAAACCCAGGTCTC-3' \\
\hline HN 101 & 3'-GACGTCCAGTTGGACGTGTCACGATGTCTGACCTTGTTTTTGGGTCCAGAG-5' \\
\hline Methylation at the heptamer ${ }^{1}$ & 1234567 \\
\hline HN 102 & 5'-CTGCAGGTCAACCTGCACGGTGCTACAGACTGGAACAAAAACCCAGGTCTC-3' \\
\hline HN 103 & 3'-GACGTCCAGTTGGACGTGCCACGATGTCTGACCTTGTTTTTGGGTCCAGAG-5' \\
\hline HN 104 & 5'-CTGCAGGTCAACCTGCA ${ }^{\mathrm{m}} \mathrm{CG}$ GTGCTACAGACTGGAACAAAAACCCAGGTCTC-3' \\
\hline HN 105 & 3'-GACGTCCAGTTGGACGT GeCACGATGTCTGACCTTGTTTTTGGGTCCAGAG-5' \\
\hline Methylation at the spacer ${ }^{2}$ & 123456789 \\
\hline HN 110 & 5'-CTGCAGGTCAACCTGCACAGTGCTACGGACTGGAACAAAAACCCAGGTCTC-3' \\
\hline HN 111 & 3'-GACGTCCAGTTGGACGTGTCACGATGCCTGACCTIGTTTTTGGGTCCAGAG-5' \\
\hline HN 112 & 5'-CTGCAGGTCAACCTGCACAGTGCTA'CGGACTGGAACAAAAACCCAGGTCTC-3' \\
\hline HN 113 & 3'-GACGTCCAGTTGGACGTGTCACGATGCCTGACCTTGTTTTTGGGTCCAGAG-5' \\
\hline Methylation at the coding region ${ }^{3}$ & $-6-5-4-321$ \\
\hline HN 140 & 5'-CTGCAGGTCCGCCTGCACAGTGCTACAGACTGGAACAAAAACCCAGGTCTC-3' \\
\hline HN 141 & 3'-GACGTCCAGGCGGACGTGTCACGATGTCTGACCTTGTTTTTGGGTCCAGAG-5' \\
\hline HN 142 & 5'-CTGCAGGTC'CGCCTGCACAGTGCTACAGACTGGAACAAAAACCCAGGTCTC-3' \\
\hline HN 143 & 3'-GACGTCCAGGC $\stackrel{\mathrm{m}}{\mathrm{m} G A C G T G T C A C G A T G T C T G A C C T T G T T T T T G G G T C C A G A G-5 '}$ \\
\hline \multicolumn{2}{|l|}{ 23-RSS ${ }^{4}$} \\
\hline HN 124 & 5'-GTGGGGACAGGGGGCCACAGTGATTCAATTCTATGGGAAGCCTTTACAAAAACCATTCT-3' \\
\hline HN 125 & 3'-CACCCCTGTCCCCCGGTGTCACTAAGTTAAGATACCCTTCGGAAATGTTTTTGGTAAGA-5' \\
\hline Methylation at the heptamer ${ }^{5}$ & 1234567 \\
\hline HN 120 & 5'-GTGGGGACAGGGGGCCACGGTGATTCAATTCTATGGGAAGCCTTTACAAAAACCATTCT-3' \\
\hline HN 121 & 3'-CACCCCTGTCCCCCGGTGCCACTAAGTTAAGATACCCTTCGGAAATGTTTTTGGTAAGA-5' \\
\hline HN 122 & 5'-GTGGGGACAGGGGGCCA'CGGTGATTCAATTCTATGGGAAGCCTTTACAAAAACCATTCT-3' \\
\hline HN123 & 3'-CACCCCTGTCCCCCGGT GCCACTAAGTTAAGATACCCTTCGGAAATGTTTTTGGTAAGA-5' \\
\hline \multicolumn{2}{|l|}{ 23-RSS standard 6} \\
\hline HN138 & 5'-GTGGGGACAGGGGTACACAGTGATTCAATTCTATGGGAAGCCTTTACAAAAACCATTCT-3' \\
\hline HN139 & 3'-CACCCCTGTCCCCATGTGTCACTAAGTTAAGATACCCTTCGGAAATGTTTTTGGTAAGA-5' \\
\hline Methylation at the heptamer ${ }^{7}$ & 1234567 \\
\hline HN 134 & 5'-GTGGGGACAGGGGTACACGGTGATTCAATTCTATGGGAAGCCTTTACAAAAACCATTCT-3' \\
\hline HN 135 & 3'-CACCCCTGTCCCCATGTGCCACTAAGTTAAGATACCCTTCGGAAATGTTTTTGGTAAGA-5 \\
\hline HN 136 & 5'-GTGGGGACAGGGGTACA"CGGTGATTCAATTCTATGGGAAGCCTTTACAAAAACCATTCT-3' \\
\hline HN 137 & 3'-CACCCCTGTCCCCATGT GeCACTAAGTTAAGATACCCTTCGGAAATGTTTTTGGTAAGA-5' \\
\hline
\end{tabular}




\section{scientific report}

sequences (Ramsden et al., 1994), the 3' D $\beta 1$ RSS may not be a special case. Although DNA methylation may regulate gene expression by altering chromatin structure to an inaccessible configuration, the possibility of a direct effect on the recombination machinery cannot be excluded.

In this study, we have found that DNA methylation inhibits the cleavage activity of the RAG 1/RAG 2 complex by two different mechanisms, depending on the position of the $\mathrm{MCpG}$ around the RSS. A novel mechanism that may directly control the regional accessibility forV(D)] recombination through DNA methylation will be discussed.

\section{RESU LTS}

To investigate the effect of $\mathrm{CpG}$ methylation on the initiation of $\mathrm{V}(\mathrm{D})]$ recombination, we tested synthetic oligonucleotide substrates containing $\mathrm{mCpGs}$ at various positions in a V(D)J cleavage assay. First, the effect of methylation within the heptamer was analysed using the sequence CACGGTG as a heptamer in place of the consensus CACAGTG (see Table 1 for a list of all oligonucleotide sequences used). This sequence was chosen because it contains a naturally occurring, unique CpG site (Ramsden et al., 1994). The single-site cleavage assay was performed in the presence of $\mathrm{M} \mathrm{n}^{2+}$, using purified core RAG 1 and RAG 2 proteins (Cuomo et al., 1996). Little difference in the cleavage efficiency was seen on changing the fourth nucleotide in the consensus heptamer from adenine to guanine (Fig. 1A, lanes 2-5), consistent with previous observations (Ramsden et al., 1996). Interestingly, hemimethylation on the top strand (the coding strand in our standard RSS orientation) markedly decreased the amounts of nick and hairpin products compared with the unmethylated control (Fig. 1A, lanes 6 and 7), indicating that a single methyl group at this position interfered with the reaction. Conversely, hemimethylation on the bottom strand (the noncoding strand) had almost no effect on $V(D)$ ) cleavage (Fig. 1A, lanes 8 and 9). Methylation on both strands markedly reduced cleavage efficiency (Fig. 1A, lanes 10 and 11) to the levels seen with top-strand methylation $(<10 \%$ of the unmethylated control). These results suggest that the $\mathrm{mCpG}$ on the top strand is responsible for this direct inhibition.

We also examined the effect of heptamer methylation in the 23-RSS. Because the mouse 3' D $\beta 1$ RSS is the only RSS that has been shown to be methylated at the heptamer in vivo, substrates were designed based on this naturally occurring 23-RSS (HN 120/121). The hairpin product was barely detected in all the 23-RSS substrates tested, consistent with the finding that certain sequences that flank cleavage sites (GC in this case) do not form hairpins easily (so-called 'bad flanks'; Cuomo et al., 1996; Ramsden et al., 1996; Fig. 1B, lanes 2-11). In fact, hairpin formation was restored when it was changed to a 'good' flanking sequence, TA (Fig. 1B, lanes 12-21). Similar to the results obtained with the 12-RSS, hemimethylation on the top strand limited cleavage (Fig. 1B, lanes 6, 7, 16 and 17), whereas hemimethylation on the bottom strand did not (Fig. 1B, lanes 8, 9, 18 and 19). Again, methylation on both strands decreased the amounts of cleavage product to the levels seen with top-strand methylation (Fig. 1B, lanes 10, 11, 20 and 21). These results show that methylation at the third cytosine of the heptamer severely affected RAG-mediated single-site cleavage.

Next, we tested the possibility that the inhibition of single-site cleavage could be overcome under coupled-cleavage reaction conditions. Thus, we analysed further the effects of heptamer methylation in the presence of $\mathrm{Mg}^{2+}$, under which condition hairpin formation requires a 12/23-RSS pair (Fig. 1C, lanes 1-4 and 13-16), as shown previously (Hiom \& Gellert, 1998). We used the 23-RSS with a good flank for these experiments. A base exchange at the fourth nucleotide of the heptamer that introduce a CpG site had no effect on the cleavage reaction (Fig. 1C, lanes 5, 6, 17 and 18). Similar to the results seen for the single-site cleavage, the production of both nicks and hairpins was severely reduced by the presence of $\mathrm{mCpG}$ in the top strand (Fig. 1C, lanes 7, 8, 19 and 20). Furthermore, $\mathrm{mCpG}$ in the bottom strand did not affect the cleavage efficiency (Fig. 1C, lanes 9, 10, 21 and 22), whereas it was markedly affected when both strands were methylated (Fig. 1C, lanes 11, 12, 23 and 24). These results indicate that heptamer methylation inhibited V(D)J cleavage even under coupledcleavage reaction conditions, suggesting that methylation at this site might controlV (D)) rearrangement under physiological conditions.

We carried out further cleavage assays on substrates containing a methylation site either within the spacer or the coding sequence, in the presence of $\mathrm{Mn}^{2+}$. For the spacer methylation, we introduced a CpG sequence at the $4 / 5$ position of the 12 -bp spacer, as this is the most common CpG site in mouse and human 12-RSSs (Ramsden et al., 1994). For the coding-region methylation, a CpG sequence was introduced at the $-6 /-5$ position from the cleavage site, which is also a common CpG site in the RSSs of the mouse Tcr gene. A base exchange of adenine to guanine at the fourth nucleotide of the 12-bp spacer that created a methylation site did not affect cleavage efficiency (Fig. 1D, lanes 2-5). In contrast with the results of heptamer methylation, spacer and coding-region methylation had no apparent effect on cleavage efficiency (Fig. 1D, lanes 6-11 and 14-19, respectively). The hairpin products derived from the substrates that contained a coding-region methylation migrated faster (Fig. 1D, lanes 12-19) due to nucleotide sequence influences on migration (Yu et al., 2002). In fact, when the substrate was labelled at the $5^{\prime}$ end of the bottom strand, the mobility of the DSB product was the same as that of the product formed from the standard substrate, HN100/101, indicating that those hairpins were formed at the expected position (data not shown). We also examined positions $7 / 8,9 / 10$ and $10 / 11$ of the spacer and $-2 /-1,-4 /-3$, $-5 /-4$ and $-11 /-10$ of the coding region for effects of methylation, but these also had no effect on $V(D)$ ) cleavage (data not shown).

Potential methylation sites at position $8 / 9$ of the nonamer ACAAAAACG, at the border of position 9 of the nonamer and the intron region $A C A A A A A C C G$, and at the first two bases of the intron region (-1/-2) flanking the nonamer, were also tested. For these substrates, no clear difference was seen in the amounts of nick and hairpin products between methylated and unmethylated substrates, indicating that methylation of these sites has no effect on the reactivity of RAG 1/RAG 2 proteins (data not shown).

In summary, these results show that a unique $\mathrm{mCpG}$ in the heptamer region severely inhibits V(D)J cleavage and this inhibition is specifically caused by a methyl group at the third cytosine of the top strand. By contrast, $\mathrm{mCpG}$ at the other sites did not directly affect the $V(D)$ ) cleavage reaction. All the positions tested for the effect of the presence of an $\mathrm{MCpG}$ are summarized in Fig. $1 \mathrm{E}$.

The reduced $V(D)$ ] cleavage due to the $m C p G$ in the heptamer region could be caused by poor binding of the RAG proteins to the substrate. Thus, we next analysed the effect of RSS methylation on the binding of RAG proteins. Reactions were carried out at $37^{\circ} \mathrm{C}$ for $30 \mathrm{~min}$ in the presence of $\mathrm{Ca}^{2+}$, which supports RAG-DNA complex formation but not RAG-mediated cleavage. Incubation of the 12-RSS standard substrate (HN 100/101) with RAG 1 alone or with both RAG 1 and RAG 2 generated a single band (Fig. 2A, lane 2), or two additional bands (Fig. 2A, lane 3; single complexes, $\mathrm{SC} 1$ and $\mathrm{SC} 2$, ), respectively. 
A

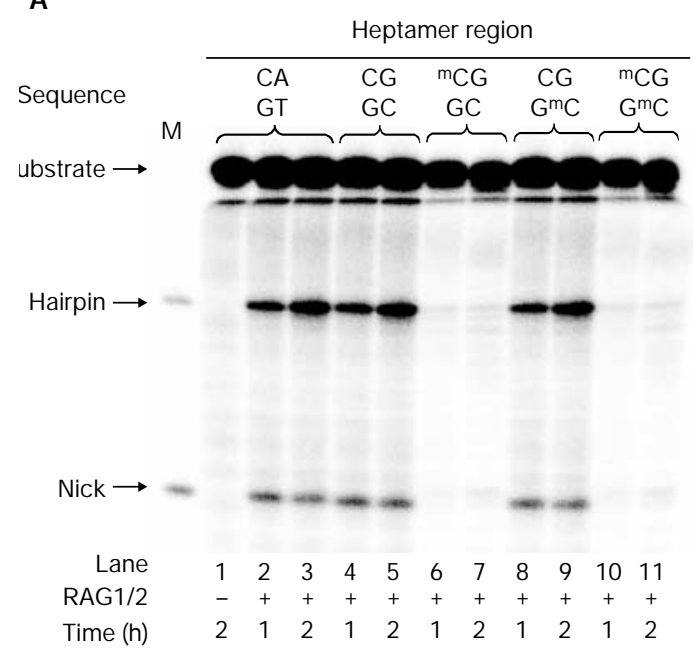

B

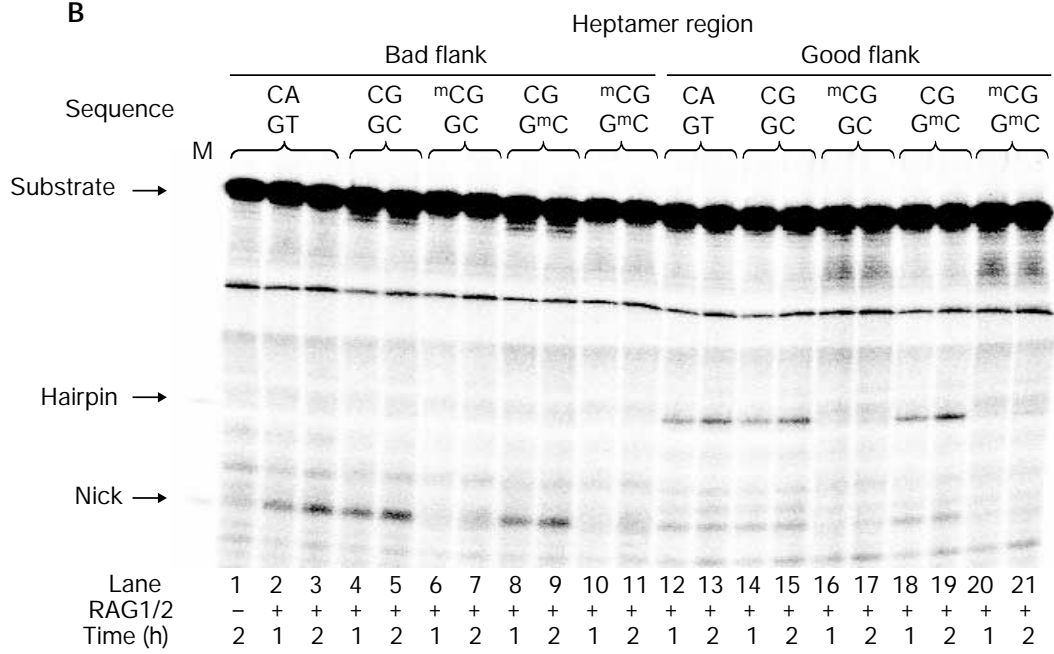

C

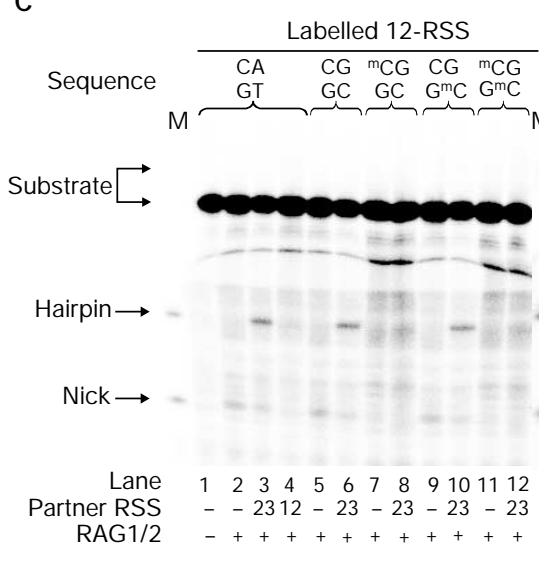

Labelled 23-RSS

$\overbrace{C A}^{G T} \overbrace{}^{C G C} \overbrace{}^{G C} \overbrace{}^{{ }^{G C G}}$

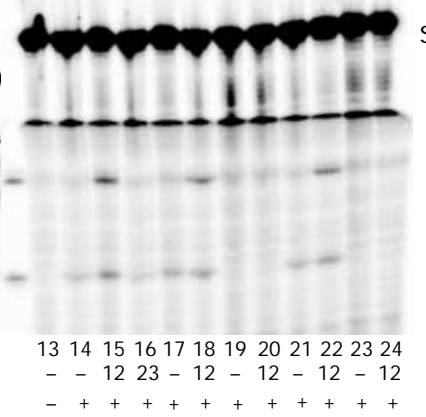

D

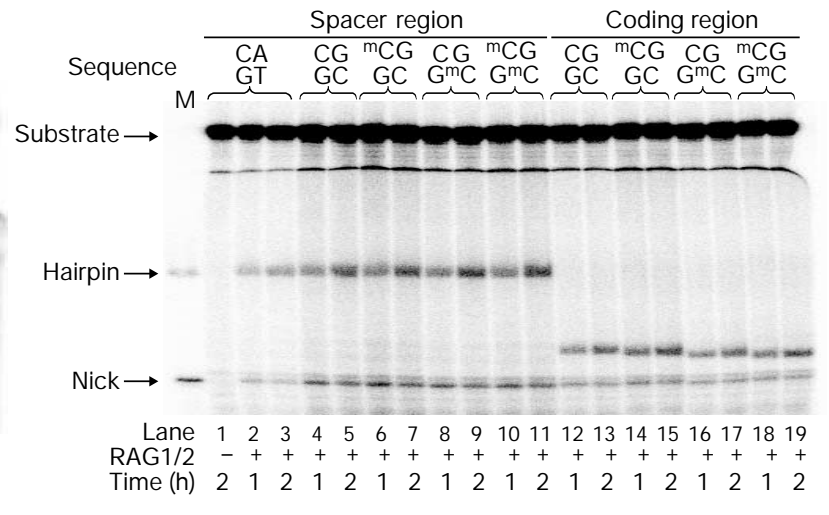

$\mathbf{E}$

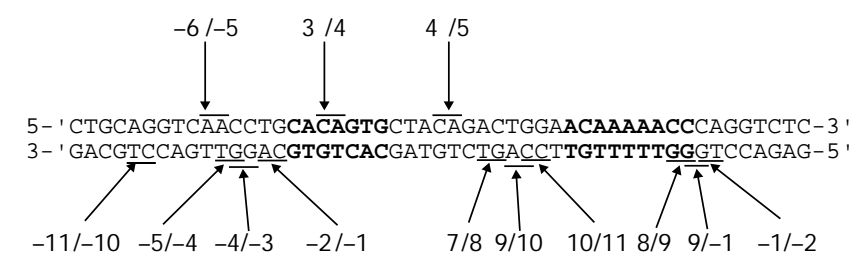

Fig. 1 |V(D)] cleavage is sensitive to the methylation at the heptamer, but not at the spacer or the coding sequence. Cleavage reactions were carried out in a mixture containing RAG1/RAG2 proteins and ${ }^{32}$ P-labelled recombination signal sequences $12-\mathrm{RSS}$ or 23-RSS, with or without methyl-CpG (mCpG). The sequences of the target dinucleotides (for example, the fourth and fifth of the heptamer) areindicated at the top of the each lane. Products were separated using a super-denaturing gel. Positions of hairpins and nicks are indicated (M). (A) Effect of methylation within the heptamer of 12-RSS. Lanes 1-3, H N 100/101; lanes 4 and 5, H N 102/103; lanes 6 and 7, H N 104/103; lanes 8 and 9, H N 102/105; lanes 10 and 11, H N 104/105. (B) Effect of methylation within the heptamer of 23-RSS. Lanes 1-3, H N 124/125; lanes 4 and 5, H N 120/121; lanes 6 and 7, H N 122/121; lanes 8 and 9, H N 120/123; lanes 10 and 11, H N 122/123; lanes 12 and 13, H 138/139; lanes 14 and 15, H N 134/135; lanes 16 and 17, H N 136/135; lanes 18 and 19, H N 134/137; lanes 20 and 21, H N 136/137. (C) Effect of methylation within the heptamer under the conditions of coupled cleavage. Left half, ${ }^{32}$ P-labelled 12-RSS (H N 100/101-H N 104/105) with or without unlabelled HN 138/139; right half, ${ }^{32}$ - -labelled 23-RSS (H N 138/139-136/137) with or without unlabelled HN 100/101. (D) Effect of methylation within the spacer (lanes 1-11) and the coding region (lanes 12-19) in the single-site cleavage. Lanes 1-3, H N 100/101; lanes 4 and 5, H N 110/111; lanes 6 and 7, H N 112/111; lanes 8 and 9, H N 110/113; lanes 10 and 11, H N 112/113; lanes 12 and 13, H N 140/141; lanes 14 and 15, H N 142/141; lanes 16 and 17, H N 140/143; lanes 18 and 19, H N 142/143. (E) Summary of the positions of mCpG tested in this study. Each dinucleotide pair tested for methylation sensitivity is indicated. Heptamer and nonamer sequences are shown in bold. Ten other mutations that weretested, but for which the results are not shown, are indicated under the nucleotide sequences. 

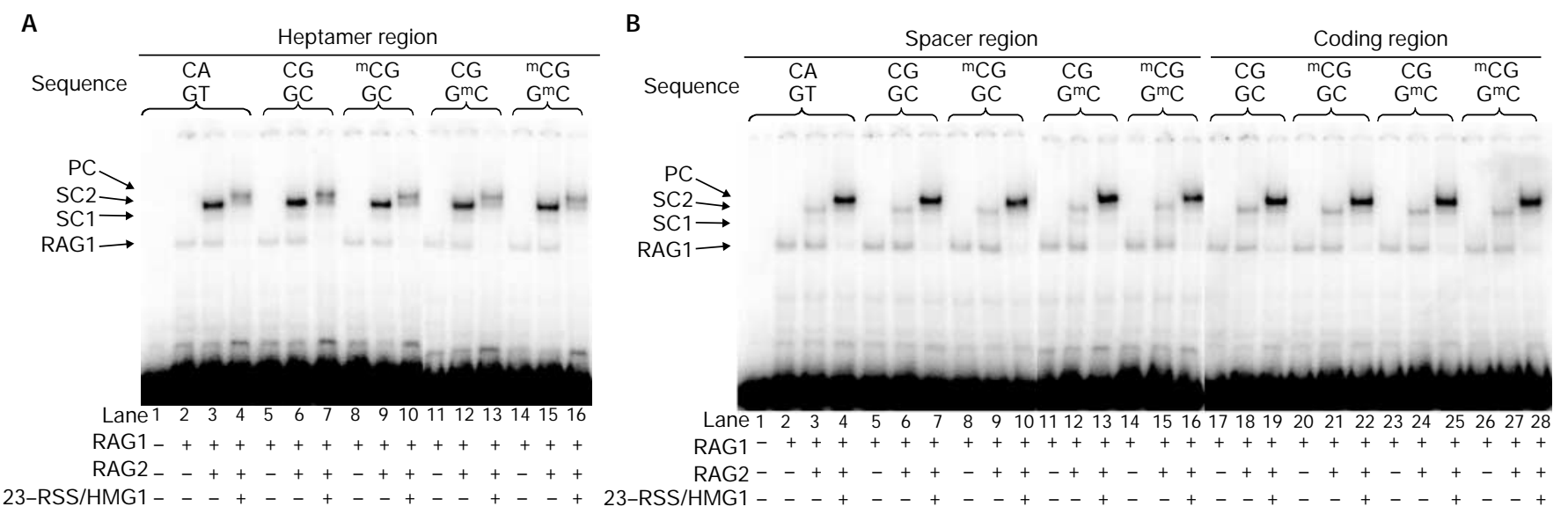

Fig. 2 | M ethyl-CpG has little effect on the binding activities of RAG1 and RAG2. Binding reactions werecarried out in buffer containing RAG1/RAG2 and 32P-labelled recombination signal sequence 12-RSS, with or without $\mathrm{mCpG}$. To detect the paired complex (PC), unlabelled 23-RSS (H N 138/139) and highmobility group 1 (HM G1) wereadded to the reaction mixtures. The complexes were separated on a native gel. Positions of each complex areindicated with arrows. Combinations of theoligonucleotide pairs are as described in the legend for Fig. 1. (A) Effect of methylation within theheptamer region. (B) Effect of methylation within the spacer region (lanes 1-16) and coding region (lanes 17-28). SC, singlecomplex.

When unlabelled standard 23-RSS was added with HMG1, a larger complex was seen, which is referred to as the PC (Fig. 2A, lane 4). Under this experimental condition, we tested the effect of RSS methylation on the formation of all the RAG 1/RAG2-DNA complexes.

The use of a substrate containing an unmethylated $\mathrm{CpG}$ sequence at the heptamer (HN 102/103) gave similar results as when the standard substrate was used (Fig. 2A, lanes 5-7). When the RSS was methylated at the heptamer region, all the expected complexes were detected, and the intensities of each band were similar to that of the unmethylated substrate (Fig. 2A, lanes 8-16). Similarly, no difference was seen between complex formation on 23-RSS with an $\mathrm{MCpG}$ at the heptamer compared with the unmethylated control (data not shown). These results indicate that an $\mathrm{mCpG}$ at the heptamer region does not affect the binding of RAG 1 and RAG2, although it severely inhibits the cleavage reaction. Furthermore, in the case of methylation at the spacer or coding regions, the binding features of the methylated substrate were the same as the unmethylated controls (Fig. 2B), consistent with no effect being shown in the cleavage assay. In conclusion, RSS methylation has little effect on the DNA binding of RAG 1 and RAG 2 . Importantly, the reduction in V(D)) cleavage of heptamer-methylated substrate is not due to the reduced binding of RAG proteins.

The $\mathrm{MCpG}$ is specifically recognized by MBD family proteins that recruit HDAC complexes, which results in the generation of an inaccessible chromatin structure. To study the function of M BD proteins in RAG-mediated cleavage of methylated substrates, the effect of the MBD protein was tested in a binding assay (Fig. 3 ). The results shown were obtained in the presence of $\mathrm{M} \mathrm{g}^{2+}$; however, similar results were obtained with $\mathrm{Ca}^{2+}$ as the divalent cation (data not shown). As it is not known which MBD family protein is involved in the regulation of the antigen-receptor genes, we used MBD core protein derived from $M B D 1$, which is highly conserved in all the MBD family members (Ohki et al., 2001). MBD core protein contains a mCpG-binding domain only, and specifically recognizes a fully methylated $\mathrm{CpG}$ (Ohki et al., 2001). More than $90 \%$ of fully methylated substrates bound to MBD (Fig. 3A, lane 2). When the RSS containing a methylated heptamer was tested, the bands representing $\mathrm{SC} 1, \mathrm{SC} 2$ and $\mathrm{PC}$ were reduced in the presence of $M B D$ (Fig. $3 A$, lanes $6-8$ ). The interference to RAG1/RAG2-DNA complex formation was not caused by nonspecific binding of MBD, as MBD had no effects on RAG complexes when an unmethylated substrate was used (Fig. 3A, lanes 14-16). The MBD band seen with the unmethylated substrates disappeared in the presence of poly $(\mathrm{dl}-\mathrm{dC})$, indicating that it was a result of nonspecific binding (data not shown). These results show that MBD inhibits RAG 1/RAG 2 complex binding to methylated substrates.

Similar effects were seen for the substrate containing an $\mathrm{mCpG}$ in the spacer or coding regions (Fig. 3B). The levels of all of the RAG 1/RAG2-DNA complexes were severely reduced in the presence of M BD (Fig. 3B, lanes 6-8 and 14-16). We also confirmed that no $V(D)$ J cleavage was seen in the presence of MBD for all the methylated substrates used in this study, including those methylated at ten other positions, shown in Fig. 1E (data not shown). In conclusion, $M B D$ proteins inhibit the initiation of $V(D)$ ) recombination by interfering with the DNA binding of the RAG1/RAG2 complex when the $\mathrm{mCpG}$ is located in or near the RSS.

\section{DISCUSSIO N}

In this study, we showed that DNA methylation negatively controls $V(D)$ J recombination in vitro, although there may be some cases not tested in this study in which RSS methylation has no effect. There are three possible mechanisms by which a CpG site could regulate V(D)] recombination. The simplest mechanism is by directly preventing RAG proteins from binding to the RSS by methylation. There are several examples in which an $\mathrm{mCpG}$ around the recognition sequence inhibits the function of a transcription factor by sterically hindering its binding. These include the binding of the transcription factors E2F, cyclic AM P response element binding protein (CREB), major late transcription factor (MLTF), AP2, C-MYC/MYN, nuclear factor- $K B$ (NF-KB), C-MYB and CCAAT-binding factor (CBF; for a review, see Mostoslavsky \& Bergman, 1997). This may therefore be a common mechanism for regulating the functions of biological factors by DNA 


\section{scientific report}

methylation. Interestingly, none of our methylated substrates inhibited RAG proteins from binding DNA. As the RAG $1 /$ RAG 2 complex makes contacts throughout the entire RSS (Fugmann et al., 2000), a small modification, such as a single $\mathrm{mCpG}$, may be tolerated.

The second mechanism is the indirect inhibition of RAG proteins from binding to the RSS by recruiting a protein factor. We showed that binding of the MBD core protein to the methylated substrate interfered with the formation of the RAG 1/RAG2-DNA complex. This indicates that methylation can indirectly control the reactivity of RAG proteins through binding of an MBD protein, independently of chromatin structure. This may be a major mechanism for regulation by methylation, as the presence of the M BD core protein affected all the methylated

\section{A}

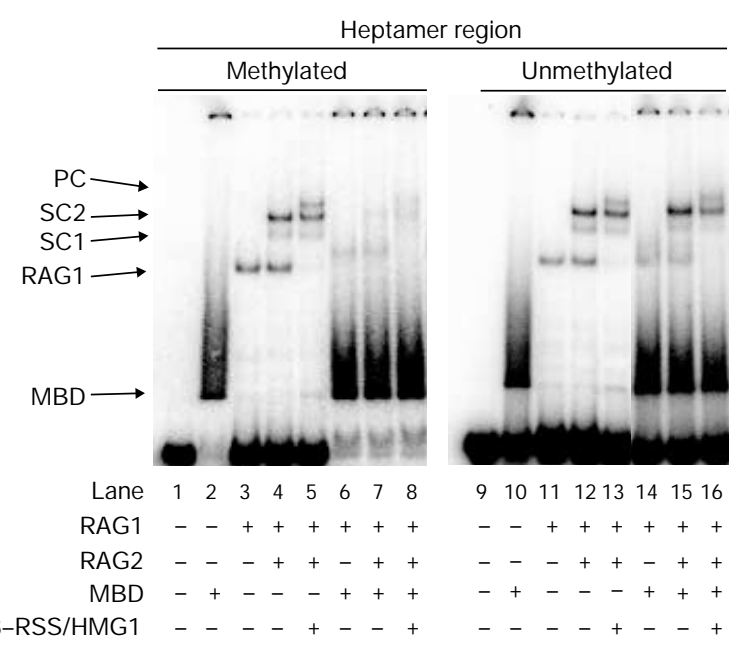

B

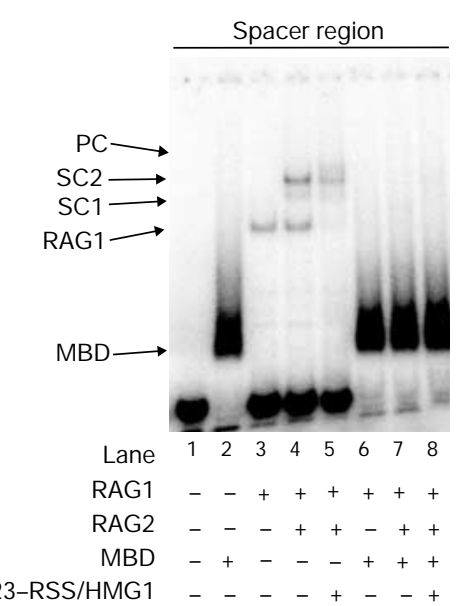

substrates tested. Because the size of a full-length M BD family protein is larger than the MBD core protein used in this study, the area around the RSS that is rendered inaccessible to RAG protein binding may span a wider region.

The third mechanism is the direct inhibition of the $V(D)$ J cleavage reaction. We found that an $\mathrm{mCpG}$ within the heptamer markedly inhibited the cleavage reaction, which was caused by a direct effect on the catalytic mechanism, without affecting the initial recognition of the site. Furthermore, we found that a single methyl group on the top strand is responsible for this effect. Figure 4 shows a predicted threedimensional structure of the consensus heptamer, on the basis of the results of Schwabe et al. (1993). In this model, the predicted position of the methyl group of methyl-cytosine on the top strand is facing the nicking surface (Fig. 4, shown in green), which may explain why the catalytic reaction was severely affected. In fact, the third nucleotide of the heptamer has been shown to make direct contact with RAG 1, as determined by ultraviolet-light cross-linking experiments (Eastman et al., 1999), which strongly suggests that this cytosine is located near the catalytic centre.

The cleavage reaction is apparently very sensitive to structural changes caused by the methylation of the top strand, but not of the bottom strand. Consensus and methylated heptamer sequences of the bottom strand are 5'-CACTGTG-3' and 5'-CAC'mCGTG-3', respectively. The position of the methyl group in methyl-cytosine occupies a spatial position similar to the thymine base of the consensus sequence. Therefore, methylation on the bottom strand would not be expected to have an effect, and our results support this view.

CpG sequences are common and appear naturally throughout the RSS. However, those in the heptamer are limited to the third cytosine of the 12-RSS. As the fourth nucleotide of the heptamer has been shown to tolerate nucleotide exchanges, such mutations might have been well conserved, as they may provide certain advantages for regulation. For example, a CpG site of mouse 3' D $\beta 1$ RSS is always

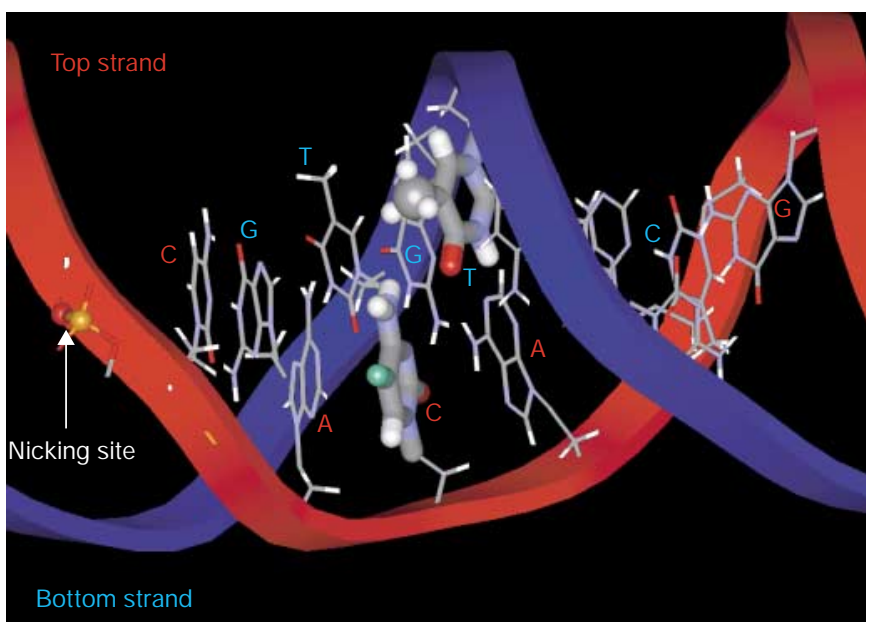

Fig. 4|Three-dimensional structure of the consensus heptamer sequence CACAGTG. The bases of the heptamer region are shown by a stick model. The phosphodiester bond where RAG1/RAG 2 introduces a nick is indicated. The third cytosine of the top strand and the fourth thymidine of the bottom strand are shown by thick lines. The site of methylation on the third cytosine is shown in green. The methyl group of the thymidine base on the bottom strand is shown as a ball model. 
demethylated when V(D)J cleavage occurs (W hitehurst et al., 2000), perhaps because demethylation is required for $V(D) J$ cleavage to proceed. The authors of this study also pointed out that the methylated allele is accessible to the $V(D)$ J recombinase, suggesting that demethylation does not al ways occur before chromatin configuration changes. Thus, the CpG sequences in or near the RSS may in some cases have an additional function that is independent of controlling accessibility though chromatin structure. Although our results, in conjunction with observations from other laboratories, suggest that specific methylation can regulate $V(D)$ ) recombination beyond chromatin regulation, the biological relevance of having this type of control remains to be explored in vivo.

\section{METHODS}

Proteins and oligonucleotide substrates. The core RAG 1 protein (amino acids 352-1,040) was purified from Escherichia coli, as described previously (Kim \& O ettinger, 1998). The core RAG 2 protein (amino acids 1-384) was purified from HeLa cells infected by recombinant vaccinia virus (McBlane et al., 1995). Bacterially expressed, purified HMG1 (amino acids 1-162) and the core MBD protein (amino acids 1-77) were provided by M.A. O ettinger and M. Shirakawa, respectively. Synthetic oligonucleotides containing 5-methyl-cytidine were purchased from Sigma. The standard HN100/101 oligonucleotides were based on the sequence of VDJ100/101 (Cuomo et al., 1996), with a base exchange to remove a pre-existing $\mathrm{CpG}$ sequence.

O ligonucleotide cleavage assays. Single-site and coupled cleavage reactions were carried out following methods described previously (Cuomo et al., 1996; Hiom \& Gellert, 1998). Briefly, for coupled cleavage, combinations of labelled and unlabelled RSSs were first mixed with RAG and $H M G 1$ proteins at $37{ }^{\circ} \mathrm{C}$ for $10 \mathrm{~min}$ in the presence of $5 \mathrm{mM} \mathrm{CaCl}$. After the pre-incubation, $\mathrm{M} \mathrm{gCl}_{2}$ was added to a final concentration of $5 \mathrm{mM}$, and the reactions were continued at $37^{\circ} \mathrm{C}$ for $30 \mathrm{~min}$. For both single-site and coupled cleavage, products were separated on a $10 \%$ polyacrylamide/0.67 $\times$ TBE denaturing gel containing $7 \mathrm{M}$ urea, $30 \%$ formamide and $12.5 \mathrm{mM}$ M OPS-KOH (pH 7.0). Gels were run at $1,800 \mathrm{~V}$ for $2 \mathrm{~h}$ and visualized using a Bio-imaging analyser, BAS2500 (Fuji). The scanned images were quantified using Image Gauge software.

DNA binding assay. Binding assays were performed as described previously ( $M$ undy et al., 2002). Binding reactions were carried out for $30 \mathrm{~min}$ at $37^{\circ} \mathrm{C}$ in the presence of $\mathrm{Ca}^{2+}$. Binding assays were also performed in the presence of $\mathrm{M} \mathrm{g}^{2+}$, but no difference was seen in the results obtained (data not shown). To elucidate the effect of MBD, $30 \mathrm{ng}$ was added to the binding reactions. Samples were separated uising $5 \%$ polyacrylamide/ $0.25 \times$ TBE gels containing $6 \%$ glycerol. Gels were run at $200 \mathrm{~V}$ at $4{ }^{\circ} \mathrm{C}$ using $0.25 \times$ TBE buffer containing $6 \%$ glycerol and were visualized as described above.

\section{ACKNOWLED GEMENTS}

Wethank M. Shirakawa for providing reagents and M .A. Oettinger for her comments on the manuscript and for providing reagents, and P. Sieh and K.B. M orshead for careful reading of themanuscript. Wearegrateful to T. Korenaga for his encouragement. This work was supported by grants from the M inistry of Education, Science, Sports and Culture of Japan (to Y.A. and Y.T.).

\section{REFEREN CES}

Bassing, C.H., Swat, W. \& Alt, F.W. (2002) The mechanism and regulation of chromosomal V(D)J recombination. Cell, 109, S15-S55

Bird, A.P. \& W olffe, A.P. (1999) M ethylation-induced repression-belts, braces, and chromatin. Cell, 99, 451-454.

Blackwell, T.K. \& Alt, F.W. (1989) M echanism and developmental program of immunoglobulin gene rearrangement in mammals. Ann. Rev. Genet., 23, 605-636.

Cherry, S.R., Beard, C., Jaenisch, R. \& Baltimore, D. (2000) V (D)] recombination is not activated by demethylation of the kappa locus. Proc. N atl Acad. Sci. U SA, 97, 8467-8472.

Cuomo, C.A., M undy, C.L. \& O ettinger, M.A. (1996) D N A sequence and structure requirements for cleavage of $V(D)]$ recombination signal sequences. Mol. Cell. Biol., 16, 5683-5690.

Eastman, Q.M., Villey, I.J. \& Schatz, D.G. (1999) D etection of RAG protein$V(D)$ J recombination signal interacts near the site of $D N A$ cleavage by UV cross-linking. Mol. Cell. Biol., 19, 3788-3797.

Fugmann, S.D., Lee, A.I., Shockett, P.E., Villey, I.J. \& Schatz, D.G. (2000) The RAG proteins and V(D)] recombination: complexes, ends, and transposition. Annu. Rev. Immunol.,18, 495-527.

Gellert, M. (2002)V(D)] recombination: RAG proteins, repair factors, and regulation. Annu. Rev. Biochem., 71, 101-132.

Hiom, K. \& G ellert, M. (1998) Assembly of a $12 / 23$ paired signal complex: A critical point in V(D)J recombination. Mol. Cell, 1, 1011-1019.

Kim, D.R. \& O ettinger, M.A. (1998) Functional analysis of coordinated cleavage in $V(D)$ J recombination. Mol. Cell. Biol., 18, 4679-4688.

M cBlane, J.F., van Gent, D.C., Ramsden, D.A., Romeo, C., Cuomo, C.A. Gellert, M . \& O ettinger, M.A. (1995) Cleavage at a V (D)] recombination signal requires only RAG 1 and RAG 2 proteins and occurs in two steps. Cell, 83, 387-395.

M ostoslavsky, R. \& Bergman, Y. (1997) D N A methylation: regulation of gene expression and role in the immune system. Biochim. Biophys. Acta, 1333, F29-F50.

M ostoslavsky, R., Singh, N., Kirillov, A., Pelanda, R., Cedar, H., Chess, A. $\&$ Bergman, Y. (1998) $\kappa$ chain monoalleic demethylation and the establishment of allelic exclusion. Genes D ev., 12, 1801-1811.

Mundy, C.L., Patenge, N., M atthews, A.G.W. \& O ettinger, M.A. (2002) Assembly of the RAG 1/RAG 2 synaptic complex. M ol. Cell. Biol., 22, 69-77.

O ettinger, M.A., Schatz, D.G. \& Baltimore, D. (1990) RAG -1 and RAG -2, adjacent genes that synergistically activate $V(D)$ J recombination. Science, 248, 1517-1523.

O hki, I., Shimotake, N., Fujita, N., Jee, J.-G., Ikegami, T., N akao, M. \& Shirakawa, M. (2001) Solution structure of the methyl-CpG binding domain of human MBD 1 in complex with methylated DNA. Cell, 105, 487-497.

Ramsden, D.A., Baetz, K. \& W U, G.E. (1994) Conservation of sequence in recombination signal sequence spacers. N ucleic Acids Res., 22, 1785-1796.

Ramsden, D.A., M cBlane, J.F., van Gent, D.C. \& Gellert, M . (1996) Distinct DNA sequence and structure requirements for the two steps of $V(D)$ ] recombination signal cleavage. EM BO J., 15, 3197-3206.

Schatz, D.G., O ettinger, M.A. \& Baltimore, D. (1989) The V(D)J recombination activating gene, RAG-1. Cell, 59, 1035-1048.

Schwabe, J.W., Chapman, L., Finch, J.T. \& Rhodes, D. (1993) The crystal structure of the estrogen receptor DNA-binding domain bound to DNA: how receptors discriminate between their response elements. Cell, 75, 567-578.

Wade, P.A. (2001) M ethyl CpG-binding proteins and transcriptional repression. Bioessays, 23, 1131-1137.

Whitehurst, C.E., Schlissel, M.S. \& Chen, J. (2000) Deletion of germline promoter PD $\beta 1$ from the TCR $\beta$ locus causes hypermethylation that impairs $D \beta 1$ recombination by multiple mechanisms. Immunity, 13, 703-714.

Yu, K., Taghva, A. \& Lieber, M.R. (2002) The cleavage efficiency of the human immunoglobulin heavy chain $\mathrm{V}_{H}$ elements by the RAG complex. J. Biol. Chem., 277, 5040-5046. 